\title{
Correction to: influenza vaccination in caregivers of childhood cancer survivors
}

\author{
In Young Cho ${ }^{1}$. Jung Eun Yoo ${ }^{2}$ Hyeonyoung Ko ${ }^{3}$ - Dong Wook Shin ${ }^{1} \cdot$ Ji Won Lee ${ }^{4} \cdot$ Hee Jo Baek ${ }^{5}$. \\ Nack-Gyun Chung ${ }^{6} \cdot$ Ki Woong Sung $^{4} \cdot$ Yun-Mi Song ${ }^{1}$
}

Published online: 3 January 2020

(C) Springer Science+Business Media, LLC, part of Springer Nature 2020

\section{Correction: Journal of Cancer Survivorship (2019) 13:993-1001. https://doi.org/10.1007/s11764-019-00825-2}

The original version of this article unfortunately contained a mistake in affiliation of the $1 \mathrm{st}, 4$ th, and corresponding author. During proof corrections stage, instead of adding new affiliation (Department of Family Medicine, Healthcare System Gangnam Center, Seoul National University Hospital, Seoul, Republic of Korea) for 2 nd author, the 1 st affiliation was replaced with the new one.

The 1st, 4th, and corresponding authors' affiliations are as follows: Department of Family Medicine, Samsung Medical Center, Sungkyunkwan University School of Medicine, Seoul, Republic of Korea.

The affiliations are corrected with this erratum.

The online version of the original article can be found at https://doi.org/ 10.1007/s11764-019-00825-2

Yun-Mi Song

yunmisong@skku.edu

1 Department of Family Medicine, Samsung Medical Center, Sungkyunkwan University School of Medicine, Seoul, Republic of Korea

2 Department of Family Medicine, Healthcare System Gangnam Center, Seoul National University Hospital, Seoul, Republic of Korea

3 Department of Family Medicine, Kangbuk Samsung Hospital, Sungkyunkwan University School of Medicine, Seoul, South Korea

4 Department of Pediatrics, Samsung Medical Center, Sungkyunkwan University School of Medicine, Seoul, Republic of Korea

5 Department of Pediatrics, Chonnam National University Hwasun Hospital, Donggu, Gwangju, Republic of Korea

6 Department of Pediatrics, the Catholic University of Korea School of Medicine, Seoul St. Mary's Hospital, Seoul, South Korea 